\title{
Regards croisés sur le bricolage et le syncrétisme*
}

Carmen Bernand, Stefania Capone, Frédéric Lenoir et Françoise Champion

\section{OpenEdition}

Journals

Édition électronique

URL : http://journals.openedition.org/assr/20727

DOI : 10.4000/assr.20727

ISSN : $1777-5825$

Éditeur

Éditions de l'EHESS

Édition imprimée

Date de publication : 1 juin 2001

Pagination : 61-66

ISBN : 2-222-96704-X

ISSN : 0335-5985

\section{Référence électronique}

Carmen Bernand, Stefania Capone, Frédéric Lenoir et Françoise Champion, «Regards croisés sur le bricolage et le syncrétisme* $»$, Archives de sciences sociales des religions [En ligne], 114 | avril-juin 2001, mis en ligne le 19 août 2009, consulté le 10 décembre 2020. URL : http://journals.openedition.org/ assr/20727 ; DOl : https://doi.org/10.4000/assr.20727

Ce document a été généré automatiquement le 10 décembre 2020.

(c) Archives de sciences sociales des religions 


\title{
Regards croisés sur le bricolage et le syncrétisme*
}

\author{
Carmen Bernand, Stefania Capone, Frédéric Lenoir et Françoise \\ Champion
}

\section{RÉFÉRENCE}

MARY (André). Le Bricolage africain des héros chrétiens. Paris, Cerf, 2000, 213 p.

\section{Détours par l'Amérique ibérique et le métissage}

1 L'ethnologue aime les sociétés pures de toute contamination occidentale. Il rejoint ainsi les missionnaires espagnols des $\mathrm{XVI}^{\mathrm{e}}$ et $\mathrm{XVII}^{\mathrm{e}}$ siècles qui condamnaient les mélanges auxquels se livraient les Indiens du Mexique et du Pérou parce qu'ils traduisaient, selon eux, l'échec de l'évangélisation. Jusqu'à une époque récente, les syncrétismes et les bricolages religieux ont constitué l'objet le moins noble de l'anthropologie. Pour la plupart des chercheurs, ces phénomènes sont superficiels et masquent des vérités que les groupes ne peuvent plus exprimer. Mais depuis quelques années, le foisonnement de nouvelles formes religieuses qui combinent, par exemple, chamanisme et religions $\mathrm{du}$ livre ou encore, l'insistance de l'Église catholique sur les valeurs spirituelles de l'inculturation, accordent aux syncrétismes une vitalité et une importance majeures.

Quelles sont les logiques qui sous-tendent ces bricolages religieux ? Le terme forgé par Claude Lévi-Strauss connut un succès considérable dans la recherche anthropologique, mais il revient à Roger Bastide de l'avoir approfondi, et d'en avoir fait un outil efficace pour comprendre les cultes afro-brésiliens, résultant de mélanges divers. Dans ce livre qui fait suite à un ouvrage sur la religion d'Éboga au Gabon, André Mary se livre à une analyse théorique du travail syncrétique, en éclairant le débat avec ses propres matériaux ethnographiques ${ }^{1}$. Le lecteur suivra avec intérêt l'exposition critique des paradigmes énoncés par Bastide, qui montrent bien que les mélanges ne sont jamais indifférenciés ni 
indifférents aux contenus sémantiques des éléments bricolés. Les pré-contraintes de la matière symbolique conservée dans la mémoire collective ou la mémoire en miettes des esclaves africains au Brésil, agissent à leur tour dans l'ajustement, toujours provisoire, des éléments constitutifs de l'ensemble.

Le paradigme de réinterprétation, c'est-à-dire de l'appropriation par la pensée indigène des contenus exogènes, est sans doute le plus connu et le plus évident, auquel s'ajoute celui de l'analogie, à partir de la sélection d'un trait signifiant du thème d'emprunt. L'exemple des différentes correspondances de Legba, qui est un des noms que l'on donne à Exu dans certains terreiros brésiliens, est significatif de la plasticité analogique des religions africaines. En tant que gardien des seuils, Legba se rapproche de saint Pierre, mais sa maîtrise du feu solaire peut également l'assimiler à saint Antoine. Enfin, pour ses caprices et ses ruses, il est associé à Lucifer. Enfin, le principe de coupure énoncé par Bastide selon lequel les Noirs du Brésil participent aux deux cultures en présence, celle catholique des Blancs et celle de leurs traditions africaines, débouche sur le compartimentage des sphères de l'existence: on peut à la fois aller à la messe, et participer à un rite de possession. En somme, la coupure est nécessaire afin d'éviter la rupture. Mais ce principe d'exclusion pose le problème de l'incompatibilité des mentalités ; il est soulevé par André Mary mais mériterait que l'on s'y attarde davantage.

Le syncrétisme religieux semble condamné à opérer entre le rapiéçage et l'inversion symbolique. C'est dans ces ajustements que les bricoleurs, que sont par exemple les prophètes africains (mais on pourrait également citer des chamanes ou même des personnalités charismatiques), jouent un rôle essentiel. André Mary nous donne un exemple concret de religion bricolée, celle du Bwiti d'Afrique équatoriale des Fang du Gabon. À la différence des prophétismes africains issus de Harris (Côte d'Ivoire, Libéria) et de Kimbangu, (Congo), ce culte est enraciné dans la tradition initiatique. Dans ses débuts, le caractère très éclaté du champ cultuel gabonais rendait impensable l'émergence d'une figure prophétique. Mais vers la fin des années 1930, à la suite d'une forte répression qui donne au mouvement ses premiers martyrs, des leaders religieux surgissent manifestant le dessein de créer une véritable religion pour les Noirs, la religion d'Éboga, et se prétendant prophètes. Mais il ne s'agit pas d'un mouvement unifié. En tout cas, l'appropriation du terme même de prophète est significative de cette tendance propre à ces religions nouvelles à puiser leur légitimité dans le champ judéo-chrétien.

5 André Mary se livre à une analyse subtile de la construction bricolée d'une religion qui doit à la fois rester fidèle à ses conceptions des différentes formes de pouvoirs et aux précontraintes que la figure du Christ impose à l'ensemble. Afin d'éviter toute superposition (contradictoire) entre forces du mal et forces bénéfiques, la création relativement récente de la catégorie intermédiaire du ngolongolo, permet de dissocier le prophète du sorcier, que la tradition plaçait au sommet de la hiérarchie des pouvoirs. Pourfendeur de la sorcellerie, le prophète exprime son pouvoir en termes de force, selon les conceptions traditionnelles. Les contradictions sont surmontées grâce à des subdivisions de cette catégorie de l'entre-deux : on distingue donc dans la classe des ngolongolo les individus "purs mais avisés» de "ceux qui font le trafic». Enfin, pour éviter de placer Jésus, désigné sous le nom de Eyene Nzame, et le premier des prophètes, dans une position subalterne par rapport au pouvoir sorcellaire, on lui confère un pouvoir d'un autre ordre, qui lui vient de Dieu et qui ne peut pas être l'équivalent de celui, suprême, détenu par le sorcier. 
6 Par un jeu complexe de reduplication et de dédoublement, le bricolage auquel se livrent les prophètes étudiés par André Mary, joue de l'ambivalence qui résulte de la confrontation de deux mondes symboliques différents. Plus encore, le travail syncrétique se révèle être un marché de dupes puisque le Noir, qui a pourtant renoncé à ses fétiches et à la sorcellerie, n'a pas obtenu en échange le pouvoir du Blanc. Mais ce constat pessimiste est dépassé par l'assurance, transmise par les prophètes, que ce pouvoir était à l'origine destiné aux Noirs, et qu'il fut détourné par les Blancs à leur avantage. Pour empêcher Jésus de révéler cette vérité aux Africains, les Blancs n'hésitèrent pas à le sacrifier. La religion Éboga permettra un jour aux Noirs de retrouver le secret du pouvoir et de renverser la situation présente.

7 Le livre d'André Mary s'achève en soulignant le parallèle que l'on peut tracer entre ces cultes syncrétiques brésiliens et africains d'une part et les formes postmodernes de mélange. Il y a là une piste prometteuse de réflexion, ouverte à de futures recherches sur les mouvements contemporains, notamment ceux qui s'inscrivent dans le sillage du New Age. À juste titre il signale (p.196) que le nouveau régime d'individualisation des croyances et des pratiques efface les tensions et les contradictions, contrairement au travail du syncrétisme qui s'est opéré dans des sociétés dominées par des valeurs collectives. Les bricolages actuels seraient donc le résultat de choix personnels en vue d'élaborer un système de valeurs «à la carte». Un syncrétisme en quelque sorte, sans pré-contraintes symboliques.

8 Quelques thèmes de discussion surgissent à la lecture de ce livre. Tout d'abord, l'auteur rejette trop vite, comme n'étant pas porteuse de paradigmes savants, ce qu'il appelle la métaphore du métissage. C'est oublier que dans toute l'Amérique ibérique, le métissage n'est justement pas une métaphore mais une réalité sociologique et politique puisque le métis n'est pas une catégorie descriptive mais un statut fiscal, déterminé par l'ambivalence raciale. Le métissage a donc une signification précise dans ce contexte-là. Mais on peut également suivre la complexité des mélanges religieux qui s'opèrent au Mexique et au Pérou au XVI ${ }^{e}$ siècle, à partir d'éléments qui n'appartiennent ni au christianisme ni aux religions indigènes, mais proviennent du paganisme antique. Les travaux de Serge Gruzinski sur les thèmes développés dans les fresques peintes par les Indiens dans les couvents, montrent par exemple, le rôle que jouent les "Métamorphoses » d'Ovide dans l'élaboration de scènes à la fois préhispaniques et chrétiennes. Le Centaure de l'église d'Ixmiquilpan et les guerriers mexicains qui l'entourent constituent un bel exemple de recomposition hybride rendue possible par la référence à des mythes de l'Antiquité. En somme, les auteurs grecs et latins facilitent la transition entre deux mondes que rien ne rapproche ${ }^{2}$.

9 Cette place à part qu'occupe le paganisme gréco-latin dans l'héritage chrétien, cette continuité que les théologiens du XVIe siècle et que les chroniqueurs établissaient entre l'Antiquité et le triomphe de l'Eglise rend difficile d'englober dans une même catégorie, celle de "paganisme", des phénomènes très différents, au nom de leur plasticité commune et de leur tolérance. En fait, les contextes historiques des syncrétismes sont fondamentaux pour comprendre leur fonctionnement, et les trois étapes de contact que distingue Bastide (p.174) sont trop générales pour être opérationnelles. Sans compter que l'ambivalence est aussi du côté du catholicisme, par exemple, dont ses propagateurs n'hésitent pas à recourir à des formes non orthodoxes de captation des Indiens. Les campagnes d'évangélisation menées par la Compagnie de Jésus sont significatives de ce brouillage. En Nouvelle-Grenade (Colombie actuelle), par exemple, les Jésuites rivalisent 
avec les chamanes muisca en utilisant les mêmes armes: miracles, visions et guérisons magiques. Il ne faut pas non plus oublier que c'est l'Église, et non pas les Indiens, qui ancre le culte mariai dans les lieux qui autrefois étaient le siège de divinités préhispaniques, tirant ainsi profit de l'ambiguïté qui en résultait.

On pourrait ajouter que le syncrétisme baroque, tel qu'il s'épanouit dans les villes des Amériques aux XVII et ${ }^{e}$ XVIII ${ }^{e}$ siècles, résulte d'une mise en spectacle des différences culturelles et religieuses. Ainsi, les Noirs, qu'ils soient esclaves ou affranchis, peuvent représenter leur musique et leurs danses dans le cadre des festivités organisées par l'Église. Il n'y a pas là de véritable résistance mais, au contraire, la manifestation de la grandeur de l'Empire et de l'Église par l'inclusion d'une mosaïque de "nations ». Cette attitude relativement tolérante par englobement de la diversité change dans la seconde moitié du XVIII e siècle, sous l'effet des politiques de modernisation et de sécularisation menées par l'État. D'où, encore une fois, la nécessité de contextualiser les phénomènes syncrétiques, aussi bien dans la conjoncture chronologique que dans le milieu géographique, étant donné que la réalité des villes n'est pas transposable au monde rural.

Une dernière remarque porte sur le monde contemporain, caractérisé, selon la belle formule d'André Mary, par la recherche d'une image de soi dans le miroir brisé des cultures du monde. Sans mettre en question l'hypothèse de l'auteur sur l'individuation des croyances, on pourrait suggérer que ces mélanges post-modernes sont une tentative de dépasser la bipolarisation chrétienne entre le Bien et le Mal, et d'affirmer l'ambiguïté des sentiments et des comportements, plus adaptée à la satisfaction des désirs personnels que la contrainte éthique. Il y a là matière à une relecture des faits, qui ne peut pas laisser insensibles tous ceux qui s'intéressent à l'anthropologie religieuse.

\section{Le syncrétisme dans tous ses états}

12 Souvent interprété comme le signe d'un manque - manque d'efficacité dans l'entreprise de conversion ou manque de résistance dans la sauvegarde de la "pureté » des traditions -, le syncrétisme constituerait en réalité, nous dit A. Mary, « l'essence de toute culture et notamment la loi de l'histoire des religions» (p.18). La question centrale que l'auteur pose est alors la suivante: comment prendre en compte cette universalité des phénomènes syncrétiques sans diluer l'objet en question et empêcher ainsi toute possibilité de conceptualisation? En réponse à cette question, A. Mary propose une idée forte: l'analyse du syncrétisme peut éclairer les procédés d'un certain type de travail symbolique, «à la manière dont les langues créoles ou pidgin peuvent éclairer pour un linguiste le problème de la formation des langues » (p. 24). Pour cela, il faut donc dégager «les logiques syncrétiques» qui sous-tendent le travail symbolique, tout en reconsidérant « la logique des pratiques de symbolisation et la place qu'elles accordent entre autres aux figures de la polysémie, de la duplicité, de l'ambivalence et du paradoxe » (p. 27).

En nous rappelant que tous les modèles d'intelligibilité des processus syncrétiques reposent sur une dialectique de la continuité et de la discontinuité, A. Mary identifie quatre paradigmes utilisés dans l'approche courante des logiques du travail syncrétique. Le premier d'entre eux est la réinterprétation. À l'instar de Herskovits et de Bastide, Mary souligne qu'il n'y a pas de syncrétisme sans réinterprétation, "c'est-à-dire sans appropriation des contenus culturels exogènes par le biais des catégories de pensée de la culture native» (p. 32). Le concept de réinterprétation est donc un concept dynamique, 
car l'élément emprunté travaille au cœur de la nouvelle structure: il laissera son empreinte sur celle-ci en la transformant inévitablement. Le travail symbolique est alors possible car l'élément emprunté est "précontraint ", en ce qu'il garde, au sein de la nouvelle structure de signification, les traces de son utilisation précédente. A. Mary montre un double mouvement à l'œuvre dans les phénomènes de réinterprétation, qui peuvent se faire dans les termes de la culture indigène ou dans ceux de la culture importée. En prenant en compte les rapports de pouvoir inhérents à cette rencontre, la réinterprétation peut ainsi être analysée comme étant le résultat de l'opposition entre culture dominante et culture dominée. En ce sens, on peut parler, comme le fait Mary, de « formes de ruse sémantique qui manient subtilement la continuité et la discontinuité en matière de changement culturel » (p. 33).

Le deuxième paradigme de la syncrétisation est l'analogie qui «pratique allègrement la ressemblance globale et l'abstraction incertaine» (p. 34) et qui introduit "la notion pseudo-savante de "correspondances" pouvant être comprise dans les termes plus élaborés d'équivalence fonctionnelle ou d'homologie structurale mais aussi dans les termes d'une mystique de la participation » (p. 35). En traitant des problèmes posés par l'identification des saints avec les orixás (les dieux du candomblé), exemple central dans la théorie des correspondances bastidienne, A. Mary parle du «caractère aveugle de la réinterprétation ", en faisant appel à la notion de " mésinterprétation constructive » ou de «malentendu productif» de M. Sahlins : « on réinterprète subrepticement sa propre culture à partir des catégories de l'autre pour aménager une sorte de rencontre providentielle entre deux cultures originelles»(p.36). Cette affirmation semblerait évacuer la notion de pré-contrainte de Lévi-Strauss, ce qui est rapidement démenti par l'auteur, lorsqu'il affirme que «le bricolage suppose également que les emprunts aux traditions importées ne s'opèrent pas dans une totale myopie structurale par rapport aux configurations de sens » (p. 41).

Le troisième paradigme de la syncrétisation est le principe de coupure, «qui permet l'alternance ou la cohabitation, chez un même individu ou au sein d'une même culture, de logiques ou de catégories en elles-mêmes incompatibles et irréductibles» (p. 37). Nous reviendrons sur ce paradigme qui est au cœur de la théorie bastidienne sur le syncrétisme et qui est lié, nous le verrons, aux trois autres.

Le quatrième et dernier paradigme repose sur la «dialectique de la matière et de la forme » (p.39), comme dirait Bastide en parlant de l'opposition entre acculturation matérielle et acculturation formelle. Bastide reprend en fait la métaphore du bricolage de Lévi-Strauss, qui considérait la matière symbolique, récupérée par le bricoleur, comme étant pré-contrainte, c'est-à-dire marquée par son usage antérieur. Cette "mémoire » inhérente à la matière symbolique devient chez Bastide une mémoire incarnée dans les corps, une mémoire collective qui se perpétue grâce aux gestes quotidiens et aux actions rituelles : «Il n’y a pas de mémoire des "éléments" en tant que tels, indépendante des marques qu'ils laissent dans les corps et les esprits des sujets déracinés » (p. 41).

\section{L'influence bastidienne}

17 Dans l'identification de ces quatre paradigmes du travail syncrétique, l'influence de Roger Bastide est évidente. A. Mary fait de l'œuvre de Bastide le témoignage d'un « cheminement exemplaire qui n'en finit pas de parcourir un à un les divers modèles d'intelligibilité disponibles sans vraiment pouvoir se fixer sur un seul» (p. 31). Il met en 
évidence l'usage désinvolte que ce dernier fait des contradictions, qui se manifestent surtout lorsqu'il s'agit d'analyser « la dialectique de la matière et de la forme » (cf. pp. 56, 102, 181, 182). D'après l'auteur, cette dialectique amorcerait, chez Bastide, une problématique "qui se cherche elle-même à travers une valse hésitation esquissant divers cas de figures et oscillant en permanence d'une position à l'autre sans qu'aucune ne soit vraiment satisfaisante » (p. 57).

Pour montrer comment ce caractère flou de la pensée bastidienne est inhérent à la théorie sur le syncrétisme qu'il a développée, nous allons concentrer notre analyse sur deux des quatre paradigmes, dont A. Mary met bien en évidence «l'instabilité profonde »: le principe de coupure et l'opposition entre acculturation matérielle et acculturation formelle. Nous verrons comment les deux autres, la réinterprétation et l'analogie, sont également à l'origine de l'élaboration de la théorie bastidienne sur le syncrétisme.

19 Selon Bastide, le principe de coupure permettrait de vivre en même temps dans deux mondes distants et incompatibles : on peut ainsi être, à la fois, un bon catholique et un adepte du candomblé. Le monde occidental et le monde africain peuvent alors coexister, sans se mélanger. Le principe de coupure implique aussi l'existence de deux types de mentalités. Pour reprendre la formulation d'A. Mary, «le principe de coupure finit dans ce cas par relever d'une logique autre, mystique ou africaine » (p.38), une logique qui serait irréductible à la nôtre. Vers la fin du livre, A. Mary essaie de lever le malentendu sur ce qu'on entend par «logique différente », en soulignant que «la pensée liante et la pensée coupante (chez Bastide) sont moins des manières différentes de penser par rapport aux nôtres que l'expression de la "philosophie dynamiste" africaine " (pp. 182-183).

Mais, cela ne remet pas en question l'idée, très forte dans les écrits de Bastide, d'une réelle irréductibilité des mentalités, mentalités non plus "plus ou moins primitives", comme dans les écrits de Lévy-Bruhl, mais intrinsèquement différentes. Bastide croyait en fait à une différence de nature entre la mentalité occidentale, moderne, et la mentalité africaine, traditionnelle; pour lui, la philosophie « dynamiste » africaine était supérieure à la culture occidentale. Cette différence permet de penser le principe de coupure: il faudra qu'un réel changement de mentalité se produise, grâce à l'acculturation formelle, pour que ces mentalités si différentes arrivent à se mélanger.

21 On pourrait regretter l'absence, dans cet ouvrage, d'une analyse plus approfondie de l'ambiguïté de fond du discours bastidien sur le syncrétisme, une analyse que A. Mary a déjà brillamment développée dans son article, «Bricolage afro-brésilien et bris-collage postmoderne $\aleph^{3}$, dans lequel il met en évidence la négation de l'idée de mélange, qui se trouve au cœur même du principe de coupure de Bastide.

\section{Le principe de coupure}

L'évolution de ce concept, depuis les années 1950 jusqu'à la mort de Bastide, n'est pas dépourvue de contradictions et de renversements inattendus. Bastide a en fait essayé de concilier la loi des participations mystiques de Lévy-Bruhl avec la loi des classifications de Durkheim, deux positions théoriques difficilement compatibles. Il a cherché à démontrer que la participation mystique n'est pas généralisée, comme l'avait affirmé Lévy-Bruhl, mais qu'elle suit un ordre, une logique bien précise. Cependant, Bastide retombe dans le piège de Lévy-Bruhl, qui postulait l'existence d'une mentalité primitive différente de la 
nôtre, lorsqu'il affirme que les classifications "primitives » ne forment pas des classes emboîtables les unes dans les autres, « comme dans notre pensée occidentale », car « elles ne permettent pas des opérations formelles ou concrètes de mécanismes opératoires $»^{4}$.

L'idée d'une incompatibilité entre ces deux mentalités sera développée dans le texte de 1958, où Bastide s'interroge sur les contraintes de la notion de coupure. Si la coupure était totale, elle rendrait impossible l'action et la pensée. Il faut donc identifier une "volonté de relier les compartiments du réel», en inventant "une dialectique du cosmos $»^{5}$. Exu devient alors cet élément dialectique, la divinité qui permet la « communicabilité des concepts classificatoires».

Le principe de coupure peut donc éclairer le problème du syncrétisme, parce que, d'un côté, il permet une "dualité sans marginalité » ${ }^{6}$ et, de l'autre, il nie le mélange : «Le terme de "syncrétisme" est juste, mais sans explication, il risque de prêter à confusion. Il ne s'agit pas de mélange, il s'agit comme dans le "rôle playing" de substitution de rôles, selon que l'on participe d'un compartiment du réel ou de l'autre $»^{7}$. Voici introduites deux notions extrêmement importantes dans la théorie bastidienne: la négation du syncrétisme en tant que mélange et la réponse à la théorie de l'homme marginal, déchiré entre deux univers, incarné par l'homme afro-américain en général et afro-brésilien en particulier. Une marginalité résolue par ce que A. Mary appelle, à juste titre, « la magie du principe de coupure » (p. 186).

On pourrait ajouter que le principe de coupure de Bastide, loin d'offrir une interprétation possible des procédés du travail syncrétique, semble au contraire faire siens les préjugés si chers à une certaine tradition anthropologique qui a toujours vu dans le mélange une marque de dégénérescence d'une pureté culturelle originelle. Avec le principe de coupure, «il n'est point question de "logiques métisses" » (p. 60). Et on pourrait aussi se demander pourquoi A. Mary ne parle pas, dans ce livre, d'un autre type de syncrétisme présent dans la théorie bastidienne, qu'il appelle « le mauvais syncrétisme », c'est-à-dire le mélange, l'hybride. En effet, pour Bastide, il n'y a pas seulement opposition entre le syncrétisme magique et le syncrétisme religieux, analysés par A. Mary, mais aussi opposition entre le syncrétisme en mosaïque, au sein duquel le principe de coupure est à l'œuvre, et le syncrétisme fusionnel, qui lui, au contraire, est condamné au mélange.

Dans le syncrétisme en mosaïque, il n'y a pas fusion, mais séparation des différents rituels ${ }^{8}$. On retrouve ici l'idée de l'existence de différents compartiments du réel formant des classes non emboîtables, comme dans le principe de coupure. Mais lorsque Bastide parle des Noirs bantou ou des "sociétés nègres ", qui auraient perdu leurs liens avec la culture originelle et la mémoire collective africaine, il est alors question d'un autre type de syncrétisme, le fusionnel, dont on ne peut discerner les éléments constitutifs. Le syncrétisme en mosaïque garde les classes séparées, le syncrétisme fusionnel les confond ${ }^{9}$ . Pour Bastide, nous rappelle A. Mary à la fin de son livre, il y a ainsi un bon et un mauvais syncrétisme (p. 204).

\section{Un syncrétisme qui n'en est pas un}

On pourrait alors se demander si Bastide a été vraiment si fidèle, comme A. Mary l'affirme (p.67), aux leçons de son maitre, G. Gurvitch, qui luttait contre ce qu'il appelait «la mystique des antinomies ». Pour sa défense, il faut dire que cette vision dualiste de la société brésilienne dominait déjà les études afro-brésiliennes lorsque Bastide arriva au Brésil. Elle était déjà présente dans les écrits du début du siècle de Nina Rodrigues, qui 
distinguait les Noirs africains (c'est-à-dire les anciens esclaves nés en Afrique), pour qui la conversion n'était qu'une " juxtaposition d'extériorités », et les Noirs créoles (les Nègres), responsables de la dégénérescence des pratiques religieuses et de leur mélange. Bastide reprend ces distinctions, formulant une théorie du syncrétisme fondée sur cette différence de nature entre les Noirs membres des candomblés traditionnels et les Noirs membres des cultes «syncrétiques». Pour les premiers, le syncrétisme n'est qu'une illusion (théorie du masque), pour les deuxièmes, il entraîne la perte des traditions africaines et la fusion des différents apports culturels. C'est-à-dire, le "mauvais » syncrétisme.

L'ensemble du travail de Bastide se structure dans ce jeu incessant entre deux pôles opposés, résultat de cette vision dualiste de la société brésilienne. Il substitue, progressivement, à la dichotomie "Africains »/«Nègres" de Nina Rodrigues (une dichotomie reproduite par Bastide lorsqu'il analyse les différents modèles d'organisation sociale dans Les Amériques noires en 1967) une autre dichotomie interne à l'objet, « Noirs nagô »/« Noirs bantou », qui entraîne à son tour une avalanche d'oppositions :

- syncrétisme en mosaïque/syncrétisme fusionnel

- pureté/dégradation

- religion/magie

- résistance/adaptation

- tradition/modernité

- civilisation religieuse africaine/idéologie de classe

- société africaine/société nègre

- continuité/discontinuité

- acculturation matérielle/acculturation formelle.

L'expression « acculturation formelle » a été formulée par Bastide à la suite des contacts qu'il a eus avec les futurs cadres africains, formés dans les universités européennes au moment de la décolonisation. Ces Africains auraient subi des transformations profondes "des structures perceptives, logiques et affectives». Bastide les accuse d'être des «intellectuels européanisés» qui chantent une Afrique «exotique» et, surtout, une Afrique «d'hommes blancs». Pour Bastide, la négritude ne serait qu'une "prise de conscience de l'Afrique par des sensibilités et des intelligences désafricanisées » et, si la négritude a échoué, c'est parce qu'elle n'a pas «cherché ses leçons » parmi les initiés aux religions africaines ${ }^{10}$. Les seuls Noirs encore " africains", ce sont les initiés aux religions traditionnelles de la diaspora, préservées sous le masque du syncrétisme. Eux, et seulement eux, peuvent devenir les véritables élites du mouvement de renouveau de la culture africaine. C'est ce que Bastide appelle «l'Africanitude», par opposition au terme négritude.

Le Brésil, et notamment le Brésil «noir et traditionnel» de Bahia, devient donc un exemple pour les élites africaines ${ }^{11}$, car la religion constitue le «foyer culturel» de la tradition africaine. Réafricaniser les élites africaines exige de renouer avec la spiritualité qui a été sauvegardée dans la diaspora. Comble du paradoxe, le colonialisme " acculture » et produit des mentalités occidentales dans des corps africains, tandis que l'esclavage sauvegarde la tradition africaine dans les pratiques religieuses. Les Africains doivent alors rechercher leurs traditions dans les communautés de culte traditionnelles qui, grâce à l'enkystement social et culturel, ont sauvegardé la vraie Afrique au Brésil. 
40 A. Mary parle d'une "ambiguïté terminologique» dans l'utilisation bastidienne du concept de forme, si forte que «la rupture épistémologique annoncée, notamment par rapport au concept classique de réinterprétation, peut s'en trouver gommée » (p. 52). En effet, la critique de Bastide à l'égard de la notion de réinterprétation visait les théories culturalistes nord-américaines et le travail de son collègue, M. Herskovits. Mais Bastide retombe dans le piège du culturalisme, lorsqu'il valorise non plus la culture en général, ou des « traits culturels » en particulier, mais la religion par rapport à la société globale. On retrouve dans ses écrits l'idée, précédemment élaborée par Herskovits ${ }^{12}$, de la religion en tant que « foyer culturel ».

41 Cette confrontation incessante avec son grand rival de l'époque amène Bastide à accuser Herskovits de perpétuer une idéologie de Blanc, selon laquelle le Noir est incapable de penser comme un Occidental. Selon Bastide, Herskovits ne s'intéressait qu'à un type de réinterprétation, celle des réalités occidentales en termes africains (par exemple, la réinterprétation du concubinage en termes de polygamie). Or, qui plus que Bastide a souligné la différence dans les structures mentales entre les Noirs et les Blancs, et surtout entre Noirs de « nature différente »? Et lorsqu'il accuse Herskovits de croire à la « double indissolubilité » des mentalités, noire et occidentale, n'est-il pas en train de critiquer sa propre théorie du masque et son principe de coupure?

42 Penser la plasticité des syncrétismes africain ou brésilien en termes de dualisme culturel sans mélange et sans déchirement offre certes une perspective séduisante. Mais A. Mary nous rappelle à juste titre qu'aujourd'hui « l'image du Noir jouant sur les deux tableaux, celui de la tradition et celui de la modernité, sans duplicité, en toute innocence, a pris le relais des représentations exotiques de l'autre culturel» (p. 192). Et il nous rappelle aussi une autre vérité : que toute tradition est syncrétique, avant de s'épurer en vieillissant. Le syncrétisme est toujours estompé par un discours traditionaliste, comme dans le Bwiti, étudié par Mary, où l'apport chrétien serait issu de la redécouverte d'une tradition originelle. Le syncrétisme est toujours l'affaire de l'Autre !

\section{Le principe de coupure et la réafricanisation}

43 Avec le principe de coupure, Bastide ouvre la voie au processus de réafricanisation, c'està-dire à l'épuration et à la légitimation des traditions. Le principe de coupure et la notion d'enkystement culturel, qui amènent à croire en l'irréalité du syncrétisme et à la théorie du masque, posent en fait les bases théoriques des mouvements actuels de lutte contre le syncrétisme et de réafricanisation. Selon la théorie du masque de Bastide, chez les Noirs « traditionnels » le syncrétisme n'a jamais été une réalité. S'il y a eu accommodation avec la civilisation dominante, elle a été "contre-acculturative», car elle n'a été qu'un simulacre pour mieux défendre la culture et la tradition africaines. La notion de syncrétisme se métamorphose en une autre notion, très répandue de nos jours au Brésil et aux États-Unis, celle d'«accommodation-résistance». Le retour aux racines et le mouvement de réafricanisation ne sont donc que de nouvelles facettes de ce syncrétisme nié, irréel. Au moins pour un certain type de Noirs, les Noirs « traditionnels » de Bahia, il n'y a jamais eu de mélange, ce qui permet aujourd'hui le retour à une «pureté » des pratiques religieuses qui passe par la lutte contre le syncrétisme ${ }^{13}$.

Ce syncrétisme feint, "contre-acculturatif», appartient au domaine de l'acculturation matérielle: "Tant que l'acculturation n'a pas pénétré jusque dans l'intérieur des mentalités, ou tant que, par suite du principe de coupure, les mentalités ne sont changées 
que dans certains domaines, politique, économique, mais non religieux, la réinterprétation se fait donc toujours à travers les valeurs, les normes, les idéaux africains $\aleph^{14}$. Le principe de coupure porte ainsi en soi la possibilité de l'effacement du syncrétisme. Il montre l'irréalité de ce phénomène, car il ne s'agit que d'un masque qui, pour reprendre l'heureuse expression d'A. Mary, n'a pas triomphé du visage.

\section{Du bricolage syncrétique au « bris-collage » postmoderne}

Le terme syncrétisme est souvent associé, nous rappelle A. Mary, à la «simple juxtaposition de croyances et de pratiques hétérogènes et contrastées ", en un mot, à une certaine « anomie » qui caractériserait les phénomènes syncrétiques (pp. 12-13). Notons que ce caractère anomique du syncrétisme, qu'il nie lorsqu'il s'agit des "logiques syncrétiques ", réapparait, sous une autre forme, à la fin du livre, où l'auteur compare le travail syncrétique dans les cultures africaines, afro-brésiliennes et océaniennes à la culture postmoderne, analysée en tant que culture « syncrétiste ».

«Si l'on croit les sociologues des nouveaux mouvements religieux américains et européens ", écrit A. Mary (p. 172), l'invention religieuse participe de la même logique de tolérance et d'ouverture que le syncrétisme. Mais les similitudes s'arrêteraient là, car dans ce qu'on appelle aujourd'hui le « retour du sacré » il n'y aurait guère de «logiques syncrétiques ». Au contraire, il serait question de mouvements religieux qui "puisent comme dans "une boîte à outils" dans les diverses traditions religieuses [...] sans souci apparent de cohérence théologique ou d'unité liturgique» (p.172). Le discours sur la postmodernité ferait alors un usage du terme « bricolage » dans lequel les pré-contraintes seraient pratiquement gommées (ibid.).

Il s'agirait alors d'une différence de nature entre, d'une part, le travail syncrétique dans les cultures africaines, afro-brésiliennes ou océaniennes, qui «se donnent comme des traditions ou des créations authentiques en quête d'unité et d'identité ", et, d'autre part, la culture postmoderne, qui serait «syncrétiste» "puisqu'elle ne se prive pas de faire l'éloge des procédés du collage et s'inscrit explicitement dans une conversion au fragmentaire, à une réalité plurielle et éclatée, consacrant la perte de toute référence aux grands récits unitaires et fondateurs » (p. 194).

Or, au début du livre (p.21), A. Mary écrivait que le syncrétisme apparaît comme un mélange incohérent lorsqu'on se limite à un point de vue «du dehors", tandis que le point de vue « du dedans » permettrait d'en souligner le caractère de création originale et de système cohérent. On ne peut alors que se demander dans quelle mesure on ne se trouverait pas ici confronté à un "point de vue du dehors ", empêchant toute découverte d'une logique inhérente au « collage post-traditionaliste ». A. Mary souligne, à juste titre, que l'apport essentiel du paradigme du bricolage est bien la notion de «prédétermination » ou de "pré-contrainte » de la matière symbolique, notion que l'on retrouve dans la théorie de Lévi-Strauss mais aussi dans la notion de mémoire collective de Bastide. La logique du syncrétisme engendre ainsi un double mouvement. Le bricoleur obéit au départ "à une logique molle, celle des "lois" de similarité et de contiguïté matérielle pour lesquelles, d'une certaine façon, tout peut être mis en rapport avec tout " (p. 81). C'est seulement après coup que les contradictions inhérentes au bricolage syncrétique entraînent une négociation dialectique qui peut provoquer des restructurations: «Le syncrétisme s'épure en vieillissant et engendre une nouvelle tradition » (ibid.). On pourrait donc penser que « bricolage », africain ou afro-brésilien, et 
«bris-collage postmoderne" sont deux phases d'un même processus et non deux phénomènes complètement différents. Une analyse approfondie des "croyances centrales et structurantes » au sein des nouveaux mouvements religieux pourrait révéler, comme le suggère $\mathrm{F}$. Champion ${ }^{15}$, l'existence de systèmes symboliques «tout à fait cohérents » qui rendraient cette opposition obsolète.

\section{Bricolage néotraditionnel et « bris-collage postmoderne »}

S'il colle bien à son terrain, le titre de l'ouvrage d'André Mary est un peu trompeur, ou trop modeste. Car si l'auteur s'attache à montrer le bricolage à l'œuvre en Afrique à travers l'exemple de la capture des héros chrétiens, ce livre est avant tout un essai brillant sur les logiques syncrétistes et tout particulièrement sur le paradigme structuraliste du bricolage. Parmi les divers paradigmes du syncrétisme, André Mary choisit en effet d'accorder une attention particulière à celui du bricolage, tel qu'il a été élaboré par Claude Lévi-Strauss et Roger Bastide. L'auteur explique clairement les deux raisons qui motivent ce choix. Tout d'abord parce que « la richesse et l'instabilité de ce paradigme permettent d'approcher au plus près du défi de la pensée que représente le travail syncrétique» (p.47). Nous ne reviendrons pas ici sur les importants développements que consacre l'auteur au concept du bricolage pour montrer sa pertinence comme paradigme du défi syncrétique. La deuxième raison invoquée concerne le «succès de la métaphore introduite en son temps par Cl. Lévi-Strauss et reprise par bien d'autres (de Certeau notamment) dans les champs les plus divers». Or André Mary souligne que ce succès "a plutôt conduit à méconnaître le modèle complexe d'intelligibilité dont il était porteur et à lui retirer des vertus heuristiques qui étaient inséparables de sa dimension technique.» (p.47). L'auteur vise ici explicitement les nombreux sociologues qui travaillent sur la modernité religieuse et qui utilisent volontiers la métaphore du bricolage pour évoquer les pratiques de mélanges et de religiosité à la carte qu'ils observent un peu partout en Occident depuis une trentaine d'années. Comme l'évoque André Mary, l'introduction du terme en sociologie religieuse est très probablement le fait de Michel de Certeau ${ }^{16}$ et il a été ensuite fortement vulgarisé par les travaux, entre autres, de Danièle Hervieu-Léger. L'auteur pointe donc une sorte de « dérive de sens » dans le réemploi du mot de bricolage par les sociologues qui travaillent sur la modernité religieuse et pose la question de la légitimité de la réutilisation d'un terme forgé dans une pensée et un contexte bien spécifique dans un cadre conceptuel et un contexte différent. C'est sur cette critique précise que portera notre lecture du livre d'André Mary.

L'auteur reconnaît qu'il existe un «air de famille» entre le syncrétisme religieux postmoderne tel qu'on peut l'observer en Europe et aux États-Unis et les syncrétismes de l'Afrique ou du Brésil actuels qui a pu conduire certains sociologues et anthropologues à "postuler l'identité profonde des logiques culturelles en présence» (p.173). Il admet également volontiers que « la praxis du bricolage qu'illustrent les faits afro-brésiliens ou les syncrétismes africains n'est pas pour autant sans pertinence pour rendre compte des nouvelles formes de syncrétismes religieux associées au fameux retour du sacré et à l'émergence de la postmodernité, mais à condition, comme dirait Claude Lévi-Strauss, qu'on soit sensible aux différences plus qu'aux ressemblances.»(p. 194). Pour bien pointer ces différences, malgré une certaine isomorphie trompeuse, l'auteur s'attache 
donc à retracer les étapes de l'élaboration du concept de bricolage dans la pensée structuraliste et à montrer combien celui-ci est devenu un paradigme clef des syncrétismes qu'on peut observer dans les cultes ou religions néotraditionnels d'Afrique ou d'Amérique. (cf. supra Stefania Capone et Carmen Bernand). De manière évidemment beaucoup plus rapide, il consacre en contre point quelques pages à la culture postmoderne et aux expressions actuelles du religieux en Occident. Il rappelle tout d'abord, à la suite de C. Lasch, R. Sennett et G. Lipovetsky ${ }^{17}$, que cette culture représente le point d'aboutissement ultime des valeurs de la modernité et la conversion à cette forme extrême de l'individualisme "narcissique ». Il s'inspire ensuite plus longuement des travaux de M. de Certeau et surtout de D. Hervieu-Léger ${ }^{18}$ pour décrire les processus de rationalisation et de subjectivisation qui minent les traditions religieuses et leur réduction à l'état de matériaux fragmentaires librement utilisés, en fonction de ses besoins du moment, par le consommateur postmoderne des biens du salut. Il souligne enfin de manière plus critique à la suite de G. Balandier ${ }^{19}$ combien cette culture narcissique et cette pratique de collage de significations fragmentaires traduit une « crise d'adhésion", qui «transforme les symboles en simulacre» (p. 195) et constitue le symptôme d'un «épuisement et d'un désespoir de sens » (p. 12).

51 André Mary arrive à la conclusion que le bricolage posttraditionnel auquel se réfèrent les sociologues se distingue du bricolage mythique tel que l'entendait Cl. Lévi-Strauss par au moins trois traits majeurs. D'une part la sortie de l'univers traditionnel encourage un «bricolage » à dominante individuelle dans un monde où les significations collectives s'estompent. Par ailleurs, malgré son syncrétisme affiché et l'hétérogénéité de ses sources, ce bricolage n'est pas travaillé par la contradiction ou l'ambivalence. "L'aspiration à la synthèse, la recherche de formation de compromis perdent de leur sens à partir du moment où le fondement ultime de ce qui fait sens est la réalisation d'un moi qui assume sa fluidité et son flottement.. » (p. 201). L'auteur avait plusieurs fois rappelé dans l'ouvrage l'importance du conflit, de la confrontation dans l'élaboration d'une nouvelle synthèse syncrétiste. Il constate au contraire que dans la quête spirituelle postmoderne « la simple juxtaposition des contraires est désormais assumée en tant que telle » et souligne qu'il s'agit même de rompre avec l'idée que « les tensions puissent être fécondes » (p. 173). Mais la différence la plus fondamentale réside dans le fait que ce bricolage postmoderne, à l'inverse du bricolage néotraditionnel, est surtout pensé en termes de « réemplois d'une matière disponible libérée des profondeurs de la conscience historique, des contraintes d'une mémoire autorisée et du respect des écarts entre systèmes de sens. » (p. 201). André Mary évoque ici la notion de " précontrainte », dont il a plusieurs fois rappelé qu'elle faisait toute la pertinence du paradigme structuraliste du bricolage : la matière symbolique qu'utilise le bricoleur est "pré-contrainte» dans la mesure où elle est marquée par son usage antérieur et conserve en partie le souvenir de sa valeur. «En reprenant un objet qui a déjà servi, le bricoleur le détourne de son usage et de sa fonction antérieure, mais les contraintes liées au marquage originel doivent être prises en compte: une cale qui devient socle reste marquée par le fait qu'elle a servie comme cale.» (p. 73). Cette notion de "pré-marquage» ou de "pré-contrainte » présuppose donc une certaine continuité de la mémoire collective. Qu'en est-il dans un monde postmoderne où une mémoire en miettes ne véhiculerait que des matériaux fragmentaires utilisés par des individus devenus amnésiques?

52 Pour toutes ces raisons, André Mary affirme l'impossibilité d'appliquer le concept structuraliste de bricolage aux pratiques occidentales contemporaines d'agencement du 
sens. « Il serait plus adapté de parler ici de « collage » posttraditionnel» (p. 201) conclut l'anthropologue. Ailleurs, l'auteur parlait de «bris-collage ». «C'est dans cet écart décisif entre le bricolage et cet avatar que représente le simple collage, que se jouent le sens ultime des créations par syncrétisme et sans doute les conditions de la survie symbolique de notre culture. » (p. 201).

Ce petit essai brillant, remarquablement écrit même s'il reste réservé à un public de spécialistes, présente un grand intérêt pour tous les chercheurs qui travaillent sur la nouvelle religiosité occidentale. La réflexion critique d'André Mary autour de la métaphorisation du concept de bricolage que nous venons sommairement de résumer, ouvre aux sociologues des religions des pistes de réflexion très fécondes. Elle peut les amener à affiner leur analyse des syncrétismes actuellement à l'œuvre dans la postmodernité et ne devrait-elle pas les engager à mieux préciser le concept de bricolage utilisé dans un contexte autre que celui des anthropologues?

Nous émettrons toutefois deux réserves à l'égard de la thèse développée ici par André Mary. On pourrait tout d'abord faire remarquer à l'auteur que l'utilisation du terme de «bricolage » dans le cadre des nouveaux syncrétismes occidentaux n'a rien d'illégitime à partir du moment où il n'est pas explicitement relié par le chercheur au modèle structuraliste. Autrement dit, rien n'empêche les sociologues d'utiliser cette métaphore dans un sens différent de celui donné par Cl. Lévi-Strauss et R. Bastide. Ainsi, lorsque D. Hervieu-Léger parle de "bricolage du sens $»^{20}$ elle ne cherche nullement à se rattacher au paradigme structuraliste, mais entend simplement évoquer cette action de mélange, d'agencement, de recomposition, que les individus effectuent à partir de matériaux divers, lesquels ne sont pas nécessairement «pré-contraints». On voit mal pourquoi la métaphore très heureuse du bricolage, laquelle évoque parfaitement les pratiques syncrétiques, devrait rester réservée à un paradigme précis ? Ce ne sera ni la première, ni la dernière fois dans l'histoire de la pensée qu'un concept ou une métaphore forgé dans un certain contexte prend un sens différent dans un autre environnement ou une autre discipline. C'est la raison pour laquelle nous ne suivons pas André Mary lorsqu'il propose de «réserver » l'utilisation du terme aux syncrétismes néotraditionnels et de parler simplement de «bris-collage » ou de "collage » pour les syncrétismes postmodernes. Mais comme nous l'avons déjà évoqué, les remarques d'André Mary pourraient inciter les sociologues à davantage affiner le concept de bricolage dans le contexte de la postmodernité et pourquoi pas d'élaborer un paradigme différent, mais tout aussi rigoureux et opérationnel que celui des structuralistes?

Notre deuxième réserve porte sur l'écart, considérable dans le propos d'André Mary, entre ce qu'on appelle faute de mieux les syncrétismes néotraditionnels et les syncrétismes postmodernes. Car on se demande parfois si l'auteur n'accentue pas un peu trop le trait traditionnel des cultes et pratiques africaines ou brésiliennes et à l'inverse le caractère postmoderne de la religiosité occidentale? Dans les deux cas de figure, il existe une tension entre tradition et modernité et on pourrait se demander si l'auteur n'exagère pas la thèse de la continuité d'un coté et celle de la rupture de l'autre? Il reconnaît luimême à propos des religions syncrétiques africaines que « l'inachèvement de la synthèse pourrait néanmoins constituer une dimension non seulement conjoncturelle mais structurelle de ces formations » (p.111). On ne saurait mieux dire des formations de la nouvelle religiosité occidentale. $\mathrm{H}$ n'est pas si sûr également que les tensions aient totalement disparus des syncrétismes occidentaux de même que les "pré-marquages ". Prenons par exemple celui de la croyance en la réincarnation qui concerne $24 \%$ des 
Occidentaux. Nos propres enquêtes de terrain montrent que certains individus n'ont effectivement aucune connaissance de la notion chrétienne de Résurrection (amnésie) à laquelle ils substituent celle de réincarnation qu'ils ne connaissent pas davantage (absence de pré-contrainte) et se forgent un concept «imaginaire» de la réincarnation totalement adapté à leur besoin. Une telle pratique conforte la thèse d'André Mary. Mais d'autres, cependant, vont avoir une certaine connaissance de la théorie orientale de la réincarnation et la réinterpréter en fonction du concept chrétien d'une âme substantielle et d'un Dieu miséricordieux. Ainsi l'élément emprunté (la théorie de la réincarnation) est non seulement "pré-contraint", mais il va également travailler au sein de la matrice d'accueil (la culture chrétienne) en vue d'une synthèse nouvelle. Nous avons ainsi rencontré des personnes encore marquées par la culture chrétienne qui, après avoir adopté dans un premier temps un peu aveuglément la théorie de la réincarnation, vivaient quelques années plus tard une véritable tension intérieure entre ces incompatibilités et tendaient à les résoudre progressivement en élaborant leur propre synthèse personnelle... laquelle peut demeurer toujours inachevée. Ici, le facteur temps est essentiel. L'auteur le reconnaît d'ailleurs très explicitement lorsqu'il parle du paradigme structuraliste: "le bricoleur extrait ou ramasse des morceaux dont la sélection obéit au départ à une logique molle, celle des «lois» de similarité et de contiguïté matérielle pour lesquelles, d'une certaine façon, tout peut être mis en rapport avec tout. C'est seulement après coup que les contradictions éventuelles entre les schèmes véhiculés par des éléments allogènes et disparates appellent une négociation dialectique qui peut provoquer des restructurations.» (p. 81) Il est sans doute trop tôt pour savoir si tous les syncrétismes occidentaux contemporains resteront, pour reprendre les catégories d'André Mary, des «bris-collages» ou deviendront des bricolages. Ces syncrétismes sont extrêmement divers, la mémoire collective n'est pas partout émiettée et de nombreuses tentatives de synthèse individuelles restent à l'œuvre. Ne vaudrait-il pas mieux nous garder de trop généraliser et de durcir le trait entre d'un coté un monde de mémoire, certes travaillé par la modernité mais encore moulé par la tradition et, d'un autre, un monde totalement amnésique soumis à l'individualisme narcissique?

56 Mais ces quelques interrogations n'enlèvent rien pour nous aux qualités de l'ouvrage d'André Mary et à son caractère extrêmement stimulant pour penser le syncrétisme contemporain.

\section{Structure et sens ; ailleurs et ici}

57 J'apprécie particulièrement la manière de travailler d'André Mary. Il ne cesse de revenir à ses auteurs de référence, Lévi-Strauss et Bastide, les faisant débattre l'un l'autre, regrettant d'ailleurs que, entre eux, la rencontre ait été « manquée » (p. 46). Il ne cesse de confronter sa propre pensée et son terrain à l'un et à l'autre. Ce faisant, le lecteur avance en confiance avec lui, et le sociologue peu pratiquant de Lévi-Strauss - ... et souvent de Bastide - peut ainsi, aussi, réviser ses classiques.

\section{L'ancrage social du travail syncrétique}

La substance essentielle du livre d'André Mary porte sur les opérations, les procédés de syncrétisation, décelables dans des corpus de croyances - Carmen Bernand et Stefania 
Capone exposent ici ces procédés. Cependant, le travail syncrétique dans sa réalité sociale effective est un travail socialement ancré. Pour parler avec les termes de Bastide, c'est à une "sociologie du bricolage" qu'André Mary veut œuvrer. Marqué par la théorie structuraliste, il en fait aussi une critique aiguë qui mérite d'être longuement citée : « Elle ne peut appréhender la discontinuité des systèmes symboliques qui structurent des cultures hétérogènes qu'en la niant sur la base d'une homologie de structure reconnue après coup (...). Pour la théorie structuraliste il ne peut y avoir plusieurs logiques en présence. Or la question que posent les formations syncrétiques (...) est celle de la confrontation entre des formes d'opposition (...) qui n'ont rien d'universelles. La logique dualiste qui sous-tend les représentations chrétiennes du bien et du mal, par exemple, n'est pas de même rang que la logique ambivalente des figures africaines et le problème de la compatibilité de ces logiques est le problème central du travail syncrétique. On aboutit au même point de rupture si, au lieu de se placer sur le plan du schématisme formel, on considérait celui des significations ou des catégories sémantiques que contiennent et ordonnent les systèmes symboliques » (p. 86).

Les procédés de symbolisation sont donc « historiquement et socialement situés » (p. 88). Cela signifie au moins trois choses. Premièrement, ils ne peuvent être compris que dans une référence serrée à la situation d'interpénétration culturelle spécifique dans laquelle ils se produisent. Par ailleurs, le travail syncrétique s'effectue dans la durée, ce qui exige une « lecture diachronique de la confrontation entre les systèmes » (p. 67), c'est-à-dire la prise en compte des différents moments d'« évolution » (le terme est d'André Mary), de transformation des corpus syncrétiques. D'autre part, le travail syncrétique est effectué par des acteurs sociaux, et tout d'abord par certains acteurs-clés, tels les prophètes d'Eboga qu'étudie précisément André Mary et dont les « histoires personnelles » (ancien catéchistes ou séminaristes) «permettent de comprendre comment plus que d'autres ils sont confrontés à des situations de double bind » (pp. 168-169).

\section{Des tensions dans la pensée d'André Mary?}

60 Avec cet ouvrage, André Mary se livre à une reprise théorique systématique de son terrain africain et de ses précédentes théorisations du syncrétisme, reprise qui apparait d'autant plus dense que l'ouvrage est concis et, de ce fait, des développements concrets de terrain peuvent parfois manquer au lecteur. Ce manque tient-il uniquement au caractère très ramassé de l'ouvrage ? Je me suis demandé si le privilège de fait accordé à la théorisation des procédés de syncrétisation sur les développements relatifs aux ancrages sociaux des syncrétismes ne tenait pas aussi à l'idée - non explicite - qui me semble sous-tendre l'objectif - explicite - d'André Mary de contribuer à une «théorie du symbolisme ». Cette idée serait que la logique des pratiques de symbolisation renvoie en dernier ressort à des procédés intellectuels inhérents à toute constitution d'un système symbolique et repérables, indépendamment de leurs ancrages sociaux, dans des corpus discursifs. Autrement dit: le projet d'André Mary me semble travaillé par la tension entre, d'une part, œuvrer à une "sociologie du bricolage », bricolage toujours "situé et contextualisé », et, d'autre part, comprendre le fonctionnement de l'esprit humain en général.

61 Cette première tension s'interpénètre avec une autre. Cette deuxième tension touche à la manière d'appréhender les systèmes symboliques: à partir de leur fonctionnement formel - sur lequel reposerait fondamentalement le sens -, ou bien à partir de leurs 
«schèmes d'interprétation" (ou «schèmes" tout court), de leurs «catégories d'entendement » ou "catégories de représentation » ou "catégories sémantiques », ou des "principes constitutifs de leur univers de sens", de leurs «significations». Toutes ces expressions, employées par André Mary et qui renvoient « directement » à du sens, ne font pas l'objet d'un examen précis, comme si André Mary ne pouvait fournir une réponse complètement assurée à la question «comment, fondamentalement, est constitué un univers de sens?».

Il peut en résulter un certain flottement comme à propos de la question de la plasticité païenne. André Mary écrit ainsi à propos de la culture africaine: «Le principe de l'addition ou de l'alternance atteste de fait, au-delà des apparences du compromis, une forme de surdité au message de l'autre. On peut même penser (...) que (ces pratiques traduisent) une négation plus profonde de l'autre que celle de la résistance et du conflit » (p. 177). Ailleurs, il parle pourtant de «la plasticité des schèmes de la culture africaine » (p. 168), de «la plasticité de la logique religieuse païenne...» (p.176). Or, à suivre la démonstration même d'André Mary il apparait bien qu'il n'y a pas - et il ne peut y avoir une plasticité des schèmes, mais qu'il existe certains schèmes qui font que peuvent «se conjuguer l'un et le multiple, le même et son contraire » (p. 176), ce qui permet d'éclairer la plasticité des figures divines païennes et leur cohabitation avec le Dieu des chrétiens ou bien, aussi, comment «le dualisme païen compose avec les hiérarchies de valeurs d'un dualisme plus radical, celui du Bien et du Mal, du Diable et du Bon Dieu » (p. 168).

Dans les développements d'André Mary, la référence directe au sens apparaît parfois fondamentale; à d'autres moments, elle semble moins utile, comme si le sens résultait avant tout de structurations formelles, comme si, peut-être, André Mary avait du mal à s'arracher à la problématique structuraliste. Je note que, dans sa conclusion, le sens réside bien, au final, dans les " catégories de l'entendement ", dans les « schèmes ".

Ces tensions que j'ai cru déceler dans la pensée d'André Mary ne lui enlève rien de son acuité. Tout au contraire, on ressent le privilège de pouvoir observer une grande pensée « en train de se faire », de se transformer.

\section{La fécondité sociologique des mises en perspective d'André Mary}

La systématisation des divers paradigmes qui permettent de comprendre l'acculturation d'un univers de sens à un autre, de comprendre la transformation des systèmes de sens, offre au sociologue un instrument d'analyse qui peut lui permettre de mieux analyser, non seulement les phénomènes "explicites » d'interactions religieuses et culturelles entre religions et cultures de "civilisations » différentes, mais aussi des recompositions et des transformations de croyances très diverses. Ainsi, par exemple, pour évoquer des phénomènes que je connais bien, de diverses nouvelles alliances actuelles entre religion et science ou entre psychologie et religion. De manière sans doute moins évidente, le recours aux divers paradigmes de l'analyse des dynamiques interculturelles pourrait également se révéler fécond pour l'analyse de ce que les sociologues désignent comme "sécularisation interne" du christianisme, c'est-à-dire son acculturation à la pensée séculière. Ce recours pourrait être d'autant plus fécond que l'acculturation du christianisme à la culture séculière qui s'est affirmée depuis le XIX siècle s'est effectuée en situation de domination et de violence symboliques de celle-ci sur celle-là. Le paradigme de la réinterprétation a toujours été très utilisé; mais on peut aussi se demander s'il n'y a pas eu quelque chose du « masque (qui) triomphe du visage » lorsque, 
par exemple, l'adoption instrumentale de pratiques démocratiques par le catholicisme l'a conduit à « mettre en péril » certains de ses principes fondamentaux ?

André Mary estime que les divers paradigmes de compréhension du travail syncrétique ont tous leur pertinence et leur intérêt. Néanmoins, le paradigme du bricolage dans la ligne de la pensée de Lévi-Strauss l'intéresse plus que les autres. Il contient en effet l'idée de pré-contrainte des matériaux bricolés. Ce qui peut être éclairant pour comprendre certaines dynamiques inter-religieuses d'aujourd'hui. Ainsi de l'« orientalisation» de l'Occident soulignée par quelques auteurs. Elle est sûrement plus limitée que le pensent ces auteurs; elle correspond néanmoins à une certaine réalité comme en témoignent, par exemple, les transformations de la catégorie « Dieu » au profit d'un « divin » impersonnel chez de plus en plus d'« Occidentaux ». L'idée de pré-contrainte peut, peut-être, expliquer de tels glissements, intervenant sur un temps relativement long, alors même que l'analyse au présent de l'adoption de croyances empruntées à l'Orient montre incontestablement la puissance des réinterprétations pour lesquelles les catégories de pensée/l'anthropologie occidentale fournissent la grille réinterprétative.

L'intérêt du paradigme du bricolage m'amène à me demander pourquoi les sociologues de la religion ont repris la métaphore du bricolage en ignorant qu'elle avait déjà été travaillée par d'aussi grands noms de l'anthropologie et de la sociologie que ceux de LéviStrauss et de Bastide. Un travail de sociologie de la connaissance sur notre discipline susceptible d'éclairer la (faible) réception de ces auteurs par les sociologues de la religion serait intéressante. En ce qui concerne certains repérages "matériels », j’ai pu satisfaire une première curiosité en me livrant à un rapide examen de la collection des Archives de Sociologie des Religions. Lévi-Strauss y est presque inconnu: seulement deux comptesrendus de ses ouvrages alors que les Archives accordaient une importance majeure, on le sait, aux recensions. La présence de Bastide a été, elle, importante mais sélective: concernant fort peu la question du syncrétisme; celle-ci intervient aux Archives essentiellement à travers deux articles importants de R. Ortiz, d'ailleurs analysés par André Mary.

68 En termes de problématique de recherche, l'ignorance des pensées de Lévi-Strauss et de Bastide sur le bricolage par les sociologues de la religion lorsqu'ils ont retrouvé la métaphore du bricolage tient au fait que leur objectif était, non pas d'étudier un travail de syncrétisation, de symbolisation, mais de comprendre la nouveauté de la situation contemporaine. Il s'agissait de comprendre, d'une part les modalités présentes de la déprise continuée des systèmes religieux institutionnels, de souligner la dérégulation et la décomposition des religions instituées, d'autre part, d'approcher le nouvel acteur social de cette dérégulation. Dans cette perspective, la métaphore du bricolage traduit une logique de "religion à la carte ", de composition personnalisée, soulignant le refus de l'individu d'aujourd'hui de prendre en bloc la religion des traditions instituées, niant donc leur logique globale spécifique. On aboutit ainsi à des compositions syncrétiques, éclectiques, bricolées : ces qualificatifs se retrouvent dans les analyses sociologiques, mais sans que l'attention se focalise vraiment sur ce point pour l'étudier de près, à la fois empiriquement et théoriquement. Lorsque ces analyses mettent en évidence certaines nouvelles manières de procéder et de croire, c'est, je crois, dans une perspective différente de celle d'André Mary. Il ne s'est pas agi pour les sociologues d'étudier positivement un travail de syncrétisation, d'analyser la transformation des significations du point de vue du travail de constitution d'un (nouvel) univers de sens. Or, c'est à partir d'une telle préoccupation qu'André Mary revisite les écrits sociologiques : ceux de Danièle 
Hervieu-Léger, sociologue de la religion, et ceux de sociologues essayistes. S'appuyant aussi sur Georges Balandier, anthropologue n'ayant pas, lui non plus, directement travaillé sur le terrain occidental, André Mary estime que, dans la société contemporaine occidentale, on a affaire non pas à du bricolage mais à du bris-collage en ce sens que «ce bricolage est surtout pensé en termes de «réemploi » d'une matière disponible libérée des profondeurs de la conscience historique, des contraintes d'une mémoire autorisée et du respect des écarts entre systèmes de sens» (p. 201), qu'il y a "refus affiché et paradoxal de toute synthèse totalisante » (p. 194).

Cette mise en perspective appelle deux questions :

70 - Une première concerne le «statut» du bricolage : s'agit-il d'une certaine pratique des acteurs sociaux conscients d'utiliser un emprunt encore marqué par l'Ailleurs dont il provient, ou s'agit-il d'un paradigme d'interprétation, signifiant que la pré-contrainte des matériaux bricolés intervient nécessairement, éventuellement, alors même que les acteurs considèrent les matériaux empruntés comme disponibles pour toutes sortes de réemploi ? De fait la pré-contrainte peut aussi travailler les compositions individuelles, "à la carte ", car l'emprunt est toujours effectué en référence à la culture importée, même si cette référence résulte d'un profond malentendu culturel.

- La seconde question porte sur le diagnostic même de collage, collage insignifiant en termes de création symbolique. Pour ne parler que des analyses des sociologues de la religion (et donc du terrain religieux, même s'il s'agit de religion décomposée), il est certain qu'André Mary a pu y trouver des arguments allant dans le sens de son diagnostic, ne serait ce que dans la métaphore du supermarché spirituel, ou dans une certaine manière de laisser affleurer une distance personnelle à l'égard des nouvelles formes religieuses «bricolées». Néanmoins, notamment à propos des Nouveaux mouvements religieux, les sociologues ont aussi souligné combien leurs adeptes sont en recherche "d'unité ", "d'harmonie », "d'authenticité ", mal à l'aise dans un monde qu'ils considèrent comme fragmenté, dépourvu de sens (bien qu'ils veuillent aussi trouver dans les nouvelles croyances des moyens efficaces pour mieux s'y insérer). En fait, je pense, à propos d'un certain éclectisme de croyances sans recherche de synthèse, que cet éclectisme porte sur des "croyances secondaires ", peu investies, d'ailleurs largement interchangeables. Il n'en va pas de même pour les croyances centrales (des catégories de pensée structurantes) définissant un croire fondamental où se jouent du «sens » - et donc des questions de sécurité existentielle, d'identité, d'unité personnelle -, de l'aspiration à un mieux-être - il s'agit de tenter de sortir d'un mal-être, voire d'une souffrance, d'une impuissance -, où se joue aussi une (ré)invention éthique après les bouleversements des années 1960-1970. Il y a, pour le moins, une cohérence subjective. Le fait qu'aujourd'hui l'initiative « religieuse » ne soit plus du côté d'une « offre » à prendre en bloc, mais du côté des individus, de leur demande, de leur "quête", de leur " recherche spirituelle », pour parler avec les termes des acteurs, le fait qu'on aboutisse ainsi à une décomposition de la religion ne veut pas forcément dire qu'on s'orienterait vers la disparition des systèmes collectifs de sens.

André Mary a pris le risque d'engager un dialogue tout à la fois interdisciplinaire et interculturel. Ce risque en vaut la peine car il contraint les sociologues de la religion à sortir de leur retranchement, à prendre davantage qu'ils le font le risque de penser la religion dé-traditionalisée pour elle-même et non pas d'abord en référence à ce qui s'est défait, à penser le genre de travail de symbolisation qu'elle effectue, à prendre le risque d'une évaluation du cours des temps présents, à considérer qu'il leur revient à eux aussi 
d'explorer très précisément la nouvelle figure de la personne en train de s'élaborer. Et le terrain de la religion, du sens, de la puissance magico-religieuse, constitue un terrain privilégié pour cette exploration.

\section{NOTES}

1. André MARY, Le défi du syncrétisme. Le travail symbolique de la religion d'Éboga (Gabon), Paris. Éditions de l'EHESS, 1999 (cf. Arch. 110.35).

2. Serge GRUZINSKI, La Pensée métisse, Paris, Fayard, 1999.

3. André MARY, "Bricolage afro-brésilien et bris-collage postmoderne ", in Paul LABURTHE-TOLRA, éd., Roger Bastide ou le réjouissement de l'abîme, Paris, L'Harmattan, 1994, pp.85-98.

4. Roger BASTIDE, "Le principe de coupure et le comportement afro-brésilien", Congrès international des américanistes de São Paulo, 1954, p. 499.

5. Roger BASTIDE, Le candomblé de Bahia (rite nagô), Paris, Mouton \& Co., 1958, p. 241.

6. Roger BASTIDE, 1954, op. cit., p. 499.

7. Roger BASTIDE, 1954, op. cit., p. 500.

8. Roger BASTIDE, Les Amériques noires, Paris, L'Harmattan, (1967) 1996, p. 75.

9. À ces deux types de syncrétisme correspondent deux types de sociétés afro-américaines (l'africaine et la nègre) et deux types de Noirs, plus ou moins «traditionnels " (les Yoruba et les Bantou). Chez les Bantou, il n'y a plus de principe de coupure, car le syncrétisme entraîne un réel changement de mentalité (Roger BASTIDE, Les religions africaines au Brésil: contribution à une sociologie des interpénétrations de civilisation, Paris, Presses Universitaires de France, 1960, p. 391).

10. Roger BASTIDE, Le prochain et le lointain, Paris, Éditions Cujas, 1970, p. 142.

11. Roger BASTIDE, 1970, op. cit., p. 227.

12. Melville Herskovits, The Myth of the Negro Past, Boston, Beacon Press, [1941] 1990.

13. Stefania CAPONE, La quête de l'Afrique dans le candomblé. Pouvoir et tradition au Brésil, Paris, Karthala, 1999.

14. Roger BASTIDE, Les religions africaines au Brésil : contribution à une sociologie des interpénétrations de civilisation, Paris, Presses Universitaires de France, 1960, p. 536.

15. Françoise CHAMPION, "La religion à l'épreuve des Nouveaux Mouvements Religieux", Ethnologie française XXX, 4, 2000, pp. 525-533.

16. Michel de CERTEAU, La culture au pluriel, Christian Bourgois, 1980 et (en collaboration avec Jean-Marie DOMENACH), Le christianisme éclaté Paris, Seuil, 1974.

17. Christopher LASCH, The culture of Narcissism, New York, New York and Co, 1979 et Gilles LIPOVETSKY, L'ère du vide, essai sur l'individualisme contemporain, Paris, Gallimard, 1983.

18. Tout particulièrement La Religion pour mémoire, Paris, Cerf, 1993.

19. Georges BALANDIER, Le détour. Pouvoir et modernité, Paris, Fayard, 1985.

20. Danièle HERVIEU-LÉGER, ibid., p. 59. 


\section{AUTEURS}

\section{CARMEN BERNAND}

Université de Paris X

\section{STEFANIA CAPONE}

Laboratoire d'Ethnologie et de Sociologie comparative - C.N.R.S.

\section{FRÉDÉRIC LENOIR}

Centre d'Études Interdisciplinaires des Faits Religieux, C.N.R.S.-E.H.E.S.S.

\section{FRANÇOISE CHAMPION}

Groupe de Sociologie des Religions et de la Laïcité - C.N.R.S. 